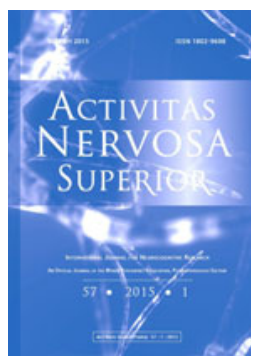

\title{
Journal
}

ANS: Journal for Neurocognitive Research Homepage:

www.activitas.org

REVIEW ARTICLE

\section{FUNCTIONAL OUTCOME IN PATIENTS WITH SCHIZOPHRENIA: THE CONCEPT AND MEASUREMENT}

\author{
Chika Sumiyoshi, ${ }^{1 *}$ Tomiki Sumiyoshi $^{2}$ \\ ${ }^{1}$ Faculty of Human Development and Culture, Fukushima University \\ Kanayagawa, Fukushima, Fukushima, Japan \\ 2Department of Clinical Research Promotion, National Center Hospital, \\ National Center of Neurology and Psychiatry, Tokyo, Japan
}

\begin{abstract}
Functional outcome has attracted interest from the perspective of interventions in patients with schizophrenia. It includes a wide range of abilities, from successful performance on neurocognitive tests to living independently in the community. The functional outcome is classified into three levels: neuropsychological performance, functional capacity, and functional performance. In the last decades, much effort has been directed into the development of assessment measures covering these domains of functional outcomes. The paper presents the concept of functional outcome and an overview of the assessments tools. Factors affecting predictability of functional outcomes and cautions in the process of translation into foreign languages are also discussed.
\end{abstract}

Key words: Schizophrenia; Functional outcome; Neuropsychological performance; Functional capacity; Functional performance

\section{INTRODUCTION}

Functional outcome is defined as social functioning/adaptation in a community, including independent living, financial management, employment, and leisure/social activities (Green et al., 2000). There has been growing concern for functional outcome in patients with schizophrenia since its inclusion in DSM-III or later editions (Burns et al., 2007). Currently, improving functional outcome in patients with schizophrenia is a highly important issue not only for clinicians, but also for governments wishing to reduce medical costs. In fact, it is widely acknowledged that people with schizophrenia make heavy use of inpatients services. The majority of those inpatients are likely to be chronically hospitalized even after remission from psychotic status, as they are incapable of participating in social and communication activities (McCrone et al., 1998). Furthermore, an increasing numbers of researchers are

${ }^{*}$ Correspondence to: Chika Sumiyoshi, Faculty of Human Development and Culture, Fukushima University,

1 Kanayagawa, Fukushima, Japan, 960-1296; e-mail: sumiyoshi@educ.fukushima-u.ac.jp

Received May 16, 2013; accepted August 20, 2014; Act Nerv Super 57(1), 1-11; ISSN-1802-9698 
oriented to develop assessment scales for the functional outcome since its inclusion into diagnostic criteria for schizophrenia in DSM-III or later versions (Burns et al., 2007).

The purpose of this paper is to present the recent concept of functional outcome in patients with schizophrenia and its measurement. Also, factors affecting predictability of functional outcome and the process of translation into foreign languages are discussed.

\section{LEVELS OF FUNCTIONAL OUTCOME}

Until the early 1990s, social/community functioning (e.g. independent living, employment, leisure/social activities) and neuropsychological performance (e.g. memory, attention, motor processing, executive functions) had been thought to reflect different capacities, and thus the functional outcome had not been necessarily linked to neuropsychological performance. However, a number of studies have reported that cognitive enhancement does help patients to obtain better social/community functioning (Green, 1996, 2006). Given this evidence, the notion of functional outcome has been re-conceptualized as a form of spectrum: a continuum from neuropsychological performance to social community functioning (Buchanan et al., 2011). It can be classified into three levels: 1) neuropsychological performance, 2) functional capacity, and 3) functional performance (Figure 1, 'functional status').

The neuropsychological performance level corresponds to basic cognitive function, which is generally assessed by neurocognitive tasks, such as memorizing letters/numbers sequences, manipulating designated signs, keeping attention to target stimulus (Figure 1, 'task/activities'). The next stage is the functional capacity, such as daily living skills in limited contexts. Functional capacity can be evaluated by role-playing (e.g. telephone communication, shopping, checking bills), or by structured interviews for patients or their care-givers. Patients may not always show the daily living skills in real situation, but at least, individuals with good functional capacity should havecompetence doing those activities (Green, Schooler et al., 2011). The highest level is the functional performance level. It refers to whether individuals are able to manage independent lives, engage in work, study, or housekeeping, enjoy leisure/social activities, and control interpersonal relationship with other community members.

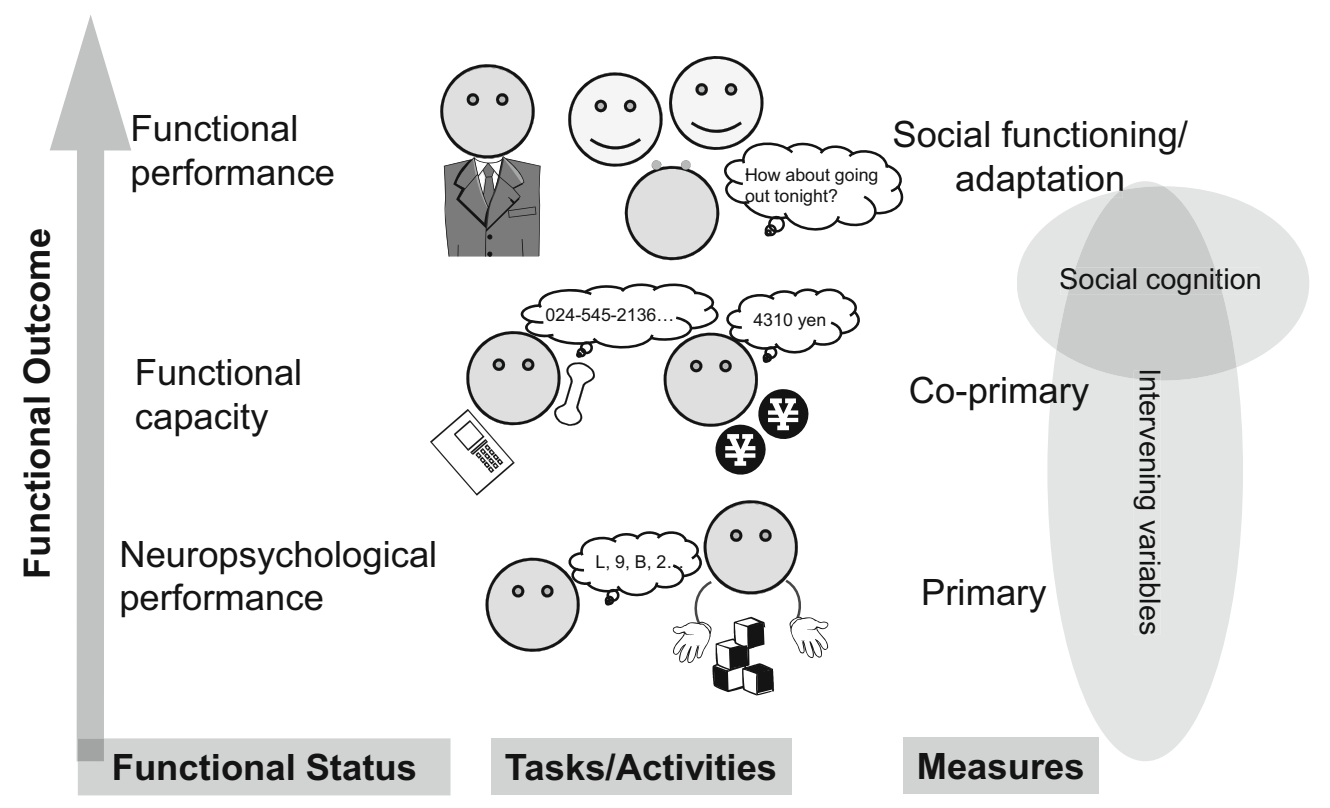

Figure 1. Levels of functional outcome. 


\section{MEASUREMENT OF FUNCTIONAL OUTCOME}

\section{1. Primary measures}

Since the early 2000s, comprehensive neurocognitive batteries tailored for schizophrenia have been developed. They include the Brief Assessment of Cognition in Schizophrenia (BACS; Keefe et al., 2004), MATRICS Consensus Cognitive Battery (MCCB; Nuechterlein \& Green, 2006), and CogState (Maruff et al., 2009; Pietrzak et al., 2009). Table 1 summarizes domains and tasks included in these 3 batteries.

The 3 batteries (Table 1) evaluate the most fundamental cognitive functions, whose recovery is assumed to be a primary target for interventions. Thus, although all they are called as primary measures (Figure 1; 'Measures'), they have different purposes or backgrounds. The principal aim of the BACS is to quickly (approximately in 30 minutes) assess cognitive deterioration in patients with schizophrenia, focusing on cognitive domains specifically impaired in the illness (Keefe et al., 2004). Contrarily, MCCB covers a wide range of cognitive domains to detect cognitive improvement by newly-developed putative cognition enhancing agents. The initial aim of the CogState Battery is to optimize sensitivity to detect cognitive change (Maruff et al., 2009). Therefore, it employs a computer-based form, covering cognitive domains of MCCB.

So far, cross-validation among the three batteries (i.e. MCCB vs. CogState vs. BACS) has not been examined. However, cross-validation between the two of them (MCCB vs. CogState, BACS vs. CogState) have been pursued with positive results (Pietrzak et al., 2009; Yoshida et al., 2011).

\section{2. Co-primary measures}

The notion of co-primary measures has been proposed through the Measurement and Treatment Research to Improve Cognition in Schizophrenia (MATRICS) project. For the approval of newly-developed anitipsychotics, the US Food and Drug Administration (FDA) emphasized that the improvement on neuropsychological performance assessed by MCCB was necessary, but not sufficient (Buchanan et al., 2011). They requested the MATRICS project committee to show the improvement on 'co-primary measures', which evaluate higher levels of functional outcome and assure the face validity of neurocognitive batteries such as MCCB. Thus, the project committee started to explore potential candidates for co-primary measures by organizing the MATRICS Co-primary and Translation (MATRICS-CT).

Ideally, co-primary measures are expected to evaluate the highest level of functional outcome, i.e. functional performance (Green, Kern et al., 2004; Green, Nuechterlein et al., 2004). However, this level of functional outcome tends to be obscured by intervening variables, such as accessibility to family/social support, availability of psycho-social rehabilitation, local economy for employment (Figure 1, the right side). Thus, functional capacity was thought to provide an alternative means for co-primary measurement (Green, Nuechterlein et al., 2008).

As previously noted, functional capacity represents the ability required in daily activities, including financial management, interpersonal communication, and handling simple documents. Whether patients can actually do those activities is not certain, as they are surrounded by stress or pressure in real world (e.g. interpersonal relationships, time limitation) (Green, Kern et al., 2004; Harvey et al., 2011). However, individuals with good functional capacity are supposed to possess competence for required activities, and they could elicit them if an appropriate and supportive environment is provided (Green, Schooler et al., 2011). 
Table 1. Neurocognitive batteries for schizophrenia.

\begin{tabular}{|c|c|c|c|c|}
\hline Batteries & Domains & Tasks/Tests & Abbreviations & Time required \\
\hline \multirow{7}{*}{$\begin{array}{c}\text { The Brief Assessment of } \\
\text { Cognition in Schizophrenia: } \\
\text { BACS }\end{array}$} & Verbal memory & List learning & & \multirow{7}{*}{ approx. $30-40 \mathrm{~min}$. } \\
\hline & Working memory & Digit sequencing task & & \\
\hline & Motor speed & Token motor task. & & \\
\hline & Verbal fluency & Category instances & & \\
\hline & & Controlled oral word association test & COWA & \\
\hline & $\begin{array}{l}\text { Attention and speed of information } \\
\text { processing }\end{array}$ & Symbol coding & & \\
\hline & Executive functions & Tower of London & TOL & \\
\hline \multirow{9}{*}{$\begin{array}{l}\text { MATRICS Consensus } \\
\text { Cognitive Battery: MCCB } \\
\text { Nuechterlein et al., } 2006\end{array}$} & Speed of processing & Trail Making Test Part A & TMT & \multirow{9}{*}{ approx.60-80 $\mathrm{min}$. } \\
\hline & & BACS-Symbol Coding & BACS SC & \\
\hline & & Category Fluency-Animal Naming & Fluency & \\
\hline & Verbal learning & Hopkins Verbal Leaning Test & HVLT-R & \\
\hline & Working memory & $\begin{array}{l}\text { Wechsler Memory Scale III-Spatial span } \\
\text { Letter Number Span }\end{array}$ & $\begin{array}{l}\text { WMS-III SS } \\
\text { LNS }\end{array}$ & \\
\hline & Reasoning and problem solving & Neuropsychological Assement Battery-Mazes & NAB Maze & \\
\hline & Visual learning & Brief Visual Memory Test (BVMT)-Revised & BVMT-R & \\
\hline & Social Cognition & $\begin{array}{l}\text { Mayer-Salovery-Caruso Emotional } \\
\text { Intelligence Test-Managing Emotion }\end{array}$ & MSCEIT $^{\mathrm{TM}}$ ME & \\
\hline & Attention and vigilance & Continuous Performance Test-Identical Pairs* & CPT-IP & \\
\hline \multirow{8}{*}{$\begin{array}{c}\text { The CogState Schizophrenia } \\
\text { Battery: CogState }\end{array}$} & Speed of processing & Detection Task* & Detect & \multirow{8}{*}{ approx. $50-70 \mathrm{~min}$. } \\
\hline & Attention and vigilance & Identification Task* & Ident & \\
\hline & Working memory & One-Back Memory Task* & One-Back & \\
\hline & & Two-Back Memory Task* & Two-Back & \\
\hline & Visual learning & Visual Learning Task* & Vis-Learn & \\
\hline & Verbal learning & International Shopping List Task* & ISLT & \\
\hline & Reasoning and problem solving & Goroton Maze Learning Task* & GMLT & \\
\hline & Social Cognition & Social Emotion Cognition Task* & SECT & \\
\hline
\end{tabular}

Note. *Computer based task.

Several test batteries/scales to measure functional capacity have been developed in recent decades. They are roughly categorized into either 'performance-based' or 'interview-based'. In the former approach, simulation (i.e. role play) of daily or social activities is conducted. In the latter, functional capacity is assessed by structured interviews of patients and caregivers. Table 2 summarizes some examples for the two approaches.

To decide which is the most appropriate as co-primary measure, the MATRICS-CT first listed selection criteria: 1) test-retest reliability, 2) utility as repeated measures, 3) relationship to functional status, 4) tolerability/practicality, 5) number of missing data, and 6) degree of correlation with cognitive performance (Green, Nuechterlein et al., 2008). Based on these criteria, several test batteries/scales were nominated to submit to the Validation of Intermediate Measures (VIM) Study. The study examined the Maryland Assessment of Social 
Competence and UPSA for performance based batteries, while SCoRS and CGI-CogS for interview-based scales.

The major findings of the VIM Study are summarized as follows (Green, Nuechterlein et al., 2008): 1) the four measures satisfy test-retest reliability; 2) correlations with neuropsychological performance are larger for performance-based batteries compared to interview-based counterparts; 3) correlations with community functioning measures are larger for performance-based batteries; 4) the number of missing data tends to be larger interviewbased scales. The study has also reported that UPSA, UPSA-B, and TABS satisfied the criteria better than other candidates. Despite the effort, no definite recommendation has been proposed so far, as to which battery/scale best meets the requirement for desirable co-primary measures (Buchanan et al., 2011).

Table 2. Co-primary measures.

\begin{tabular}{|c|c|c|c|c|}
\hline Form & Batteries/Scales & Abbreviations & Developers & Notes \\
\hline \multirow{4}{*}{ Perfroamce-based } & Maryland Assessment of Social Competence & & Bellack, et al., 1994 & \\
\hline & Test of Adaptive Behavior in Schizophrenia & TABS & Velligan et al., 2007 & Brief version is available \\
\hline & Independent Living Scales & ILS & Loeb, 1996 & \\
\hline & $\begin{array}{l}\text { University of California at San Diego Performance- } \\
\text { Based Skills Assessment }\end{array}$ & UPSA & Patterson, et al., 2001 & $\begin{array}{l}\text { Brief version is available } \\
\text { (UPSA-B; Mausbach et al., 2007) }\end{array}$ \\
\hline \multirow{3}{*}{ Interview-based } & Schizophrenia Cognition Rating Scale & SCoRS & Keefe et al., 2006 & \\
\hline & Clinical Global Impression of Cognition of Schizophrenia & CGI-CogS & Ventura et al., 2008 & \\
\hline & The Cognitive Assesment Interview & CAI & Bielder et al., 2008 & Developed from CGI-Cogs \\
\hline
\end{tabular}

\subsection{Measures for functional performance}

Measures for functional performance generally include the evaluations of social functioning (independent living, recreation, employment), social adjustment/adaptation (conforming to social expectations or cultural norms, keeping interpersonal relationship) (Burns et al., 2007). Research has been conducted vigorously to evaluate the utility of functional performance measures due, partly, tothe inclusion of the functional outcome in the DSM-III or later versions. DSM-IV-TR, for example, defines social functioning as one of the diagnostic criteria for schizophrenia. It covers three domains: work/study, interpersonal relations, and self-care (Burns et al., 2007). To meet the need for reliable information for measures of social functioning, an extensive review was conducted (Burns et al., 2007). Table 3 presents the 20 most-frequently-used scales in studies for schizophrenia.

\subsection{Predictability of functional performance}

The MATRICS-Psychonomic and Standardization Study (MATRICS-PASS) has designated a new scale, Modified Social Functioning Scale/Social Adaptation Scale for MATRICS-PASS (Modified SFS/SAS for MATRICS-PASS), for the validation study of MCCB. This scale is composed of the Social Functioning Scale (SFS; Birchwood et al., 1990) (Figure 2, left) and Work Outcome section from the modified SAS (Social Adaptation Scale; Subotnik et al., 2008) (Figure 2, right), and covers a wide range of functional performance. SFS and SAS were listed the top 10 of the most frequently used scales in clinical trials (Table 3).

Principal component analysis was conducted on Modified SFS/SAS for MATRICS-PASS data, and 3 components (i.e. Work, Independent living, and Social domains of everyday functioning) were extracted. To examine the relationship with $\mathrm{MCCB}$, correlations were 
estimated between each component and the 10 tasks in MCCB. As the result, none of combinations showed significant correlations, although the work tended toy ield larger correlations with MCCB tasks (Nuechterlein \& Green, 2006). The results suggest the difficulty of predicting functional performance based on neurocognitive performance measures, (at least in the USA). The issue is discussed in the later section.

Table 3. The frequency of use of scales for the assessment of social functioning in schizophrenia studies.

\begin{tabular}{|c|c|c|}
\hline & $\begin{array}{l}\text { Schiz ophrenia studies using } \\
\text { social functioning as an outcome } \\
\text { measure }\end{array}$ & $\begin{array}{l}\text { Rando mized contr olle d tr ials of } \\
\text { antipsychotic agents }\end{array}$ \\
\hline Functioning Scale & Frequency & Frequency \\
\hline $\begin{array}{l}\text { Global Assessment of Functioning } \\
\text { (GAF) scale }\end{array}$ & 58 & 2 \\
\hline Global Assessment Scale (GAS) & 39 & 0 \\
\hline Social Functioning Scale (SFS) & 37 & 2 \\
\hline $\begin{array}{l}\text { Social and Occupational Functioning } \\
\text { Assessment Scale (SOFAS) }\end{array}$ & 20 & 4 \\
\hline Disability Assessment Scale (DAS II) & 20 & 0 \\
\hline $\begin{array}{l}\text { Strauss-Carperter L evel of } \\
\text { Functioning scale (SC-LOF) }\end{array}$ & 25 & 2 \\
\hline Social Açustment Scale-II (SAS-II) & 19 & 1 \\
\hline Social Behaviour Schedule (SBS) & 16 & 0 \\
\hline Role Functioning Scale (RFS) & 10 & 0 \\
\hline $\begin{array}{l}\text { Groningen Social Dis ability Schedule } \\
\text { (GSDS) }\end{array}$ & 8 & 0 \\
\hline $\begin{array}{l}\text { Medical Outcomes Study Short-Form } \\
36 \text { Health Survey (SF-36) }\end{array}$ & 6 & 3 \\
\hline $\begin{array}{l}\text { Scale for Social Adaptive Fumctioning } \\
\text { Evaluation (S.AFE) }\end{array}$ & 6 & 0 \\
\hline $\begin{array}{l}\text { Miltinomah Community Ability Scale } \\
\text { (MCAS) }\end{array}$ & 6 & 0 \\
\hline Life Skills Profile (LSP) & 6 & 0 \\
\hline $\begin{array}{l}\text { Interview Schedule for Social } \\
\text { Interaction (ISSI) }\end{array}$ & 5 & 0 \\
\hline $\begin{array}{l}\text { Assessment of Interpersonal Problem- } \\
\text { Solving Skills (AIP SS) }\end{array}$ & 5 & 0 \\
\hline Community Adjustment Form (C.AF) & 4 & 0 \\
\hline $\begin{array}{l}\text { Personal and Social Perform ance } \\
\text { (PSP) scale }\end{array}$ & 3 & 3 \\
\hline
\end{tabular}

Note. The table is reproduced from Table 1 in Burns, T., \& Patrick, D. (2007). Acta Psychiatrica Scandinavica, 116, 403-418, with the permission from the publisher. Refer to Burns (2007) for the details of listed scales. 


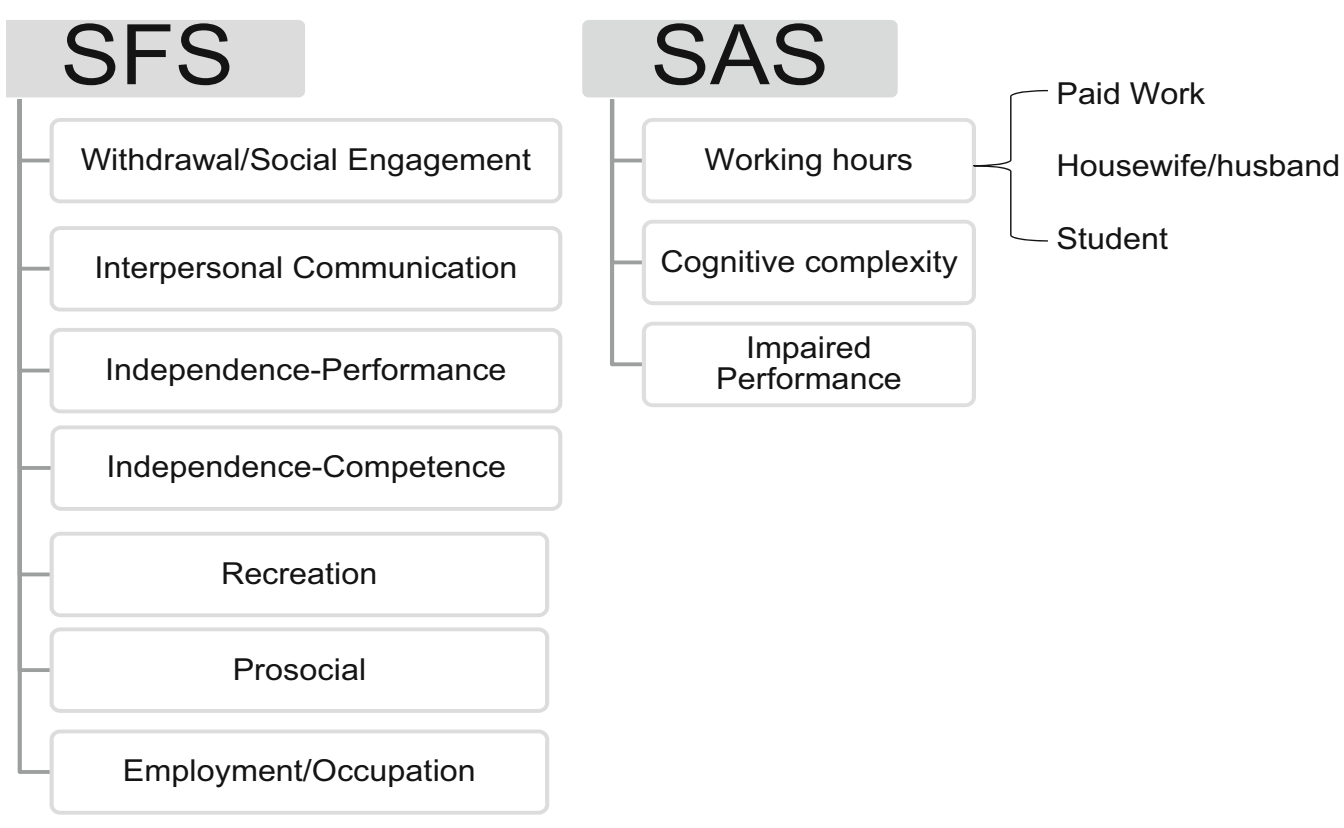

Figure 2. Modified SFS/SAS for MATRICS-PASS.

\section{INTERVENING VARIABLES AND SOCIAL COGNITION: THE RELATIONS TO FUNCTIONAL OUTCOME}

Neurocognitive assessment per se may not be sensitive enough to predict functional outcome in patients with schizophrenia. As previously noted, this is partly due tointervening variables, which are intrinsic to the community environment. They include an employment rate in a resident area, availability of social service/support, accessibility to psychosocial education, workplace environment. For instance, even if an individual could exhibithigher achievement onneuropsychological tasks/tests, she/he may not beemployed due to arecession in her/his residential area. Contrarily, even if a person is not neuropsychologically fully recovered, he/she may yet be able to work given the better recruitment and sufficient support by others.

The effect of intervening variables may vary across culture or society. In fact, unlike the finding in the US study (Nuechterlein \& Green, 2006), our preliminary study conducted in Japan has revealed a significant correlation between neuropsychological performance (MCCB score) and functional performance (SFS total score from Modified SFS/SAS for MATRICSPASS_J) (Sumiyoshi, 2011) (Figure 3). The result indicates that the former predict the latter in a certain degree, at least in Japan. Our observation suggests that relatively uniform society like Japan, improvement on basic cognitive function is more closely linked with better social/community functioning compared to the multi-cultural/socio-economical society like the US.

Another potential factor attenuating the predictability of functional outcome is social cognition, which is assumed to be acting as a mediator between neurocognitive performance and functional performance. It has been claimed that better social cognition enhances social activities in patients (Green, Bearden et al., 2011; Horan et al., 2011). Social cognition generally refers to mental operations that underlie social interactions, including perceiving facial expressions, inferring and interpreting belief or intention of others, and managing selfemotion (Green, Bearden et al., 2011). 


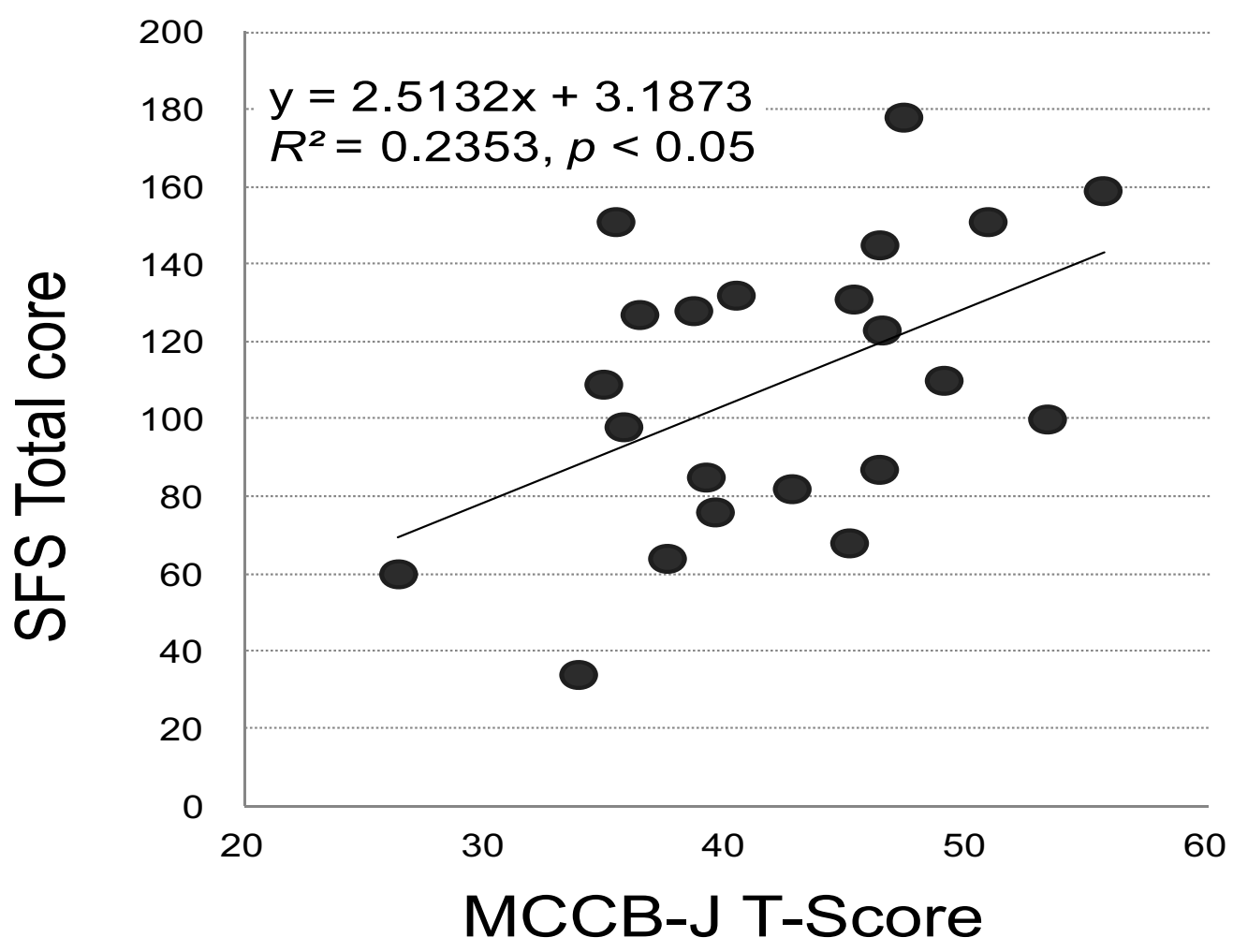

Figure 3. Regression analysis for neurological performance (MCCB) and functional performance (SFS total score from Modified SFS/ SAS for MATRICS-PASS_J).

Despite recent intensive research on social cognition (for the review, Couture et al., 2006), the internal operations for social cognition has not been fully clarified; whether social cognitive processes are independent or overlapped with neurocognitive processes is a matter of debate (Green, Penn et al., 2008). If the latter is true, social cognition is not a mediator between neurocognitive performance and functional performance. Rather, social cognition may be enhanced by the recovery of neurocognitive performance in that case. In fact, a recent study (Fanning et al., 2012) has reported that the percentage of patients with impaired neurocognitive functions but without deficits in social cognition is less than $1 \%$, while the opposite (spared neurocognitive function and impaired social cognition) is considerably higher $(25 \%)$. Furthermore, a large portion of improvement of social cognition $(83 \%)$ is explained by enhancement of neuropsychological performance (Vauth et al., 2004). These findings indicate that enhancing neuropsychological performance is essential for ameliorating social cognition (Fanning et al., 2012), and, consequently, for restoring functional outcome.

\section{INTERNATIONAL VERSIONS}

Most batteries/scales listed in Table 1 and Table 2 have been implemented in various languages other than English. $\mathrm{MCCB}$, for example, was intended to be an international standard of neurocognitive test battery for schizophrenia. It has been introduced in India, South America, China, and other Asian countries, as well as various European countries (e.g. Spain, Italy, and France) (Harvey et al., 2010). Likewise, measures of functional capacity and functional performance have been introduced into European and Asian countries. In Japan, 
for example, BACS (BACS_J; Kaneda et al., 2007), CogState (CogState_J; Yoshida et al., 2011), and SFS (SFS_J; Nemoto et al., 2008) have been translated and validated. MCCB (MCCB_J), UPSA-B (UPSA-B_J;Sumiyoshi et al., 2011), and Modified SFS/SAS for MATRICS-PASS (Modified SFS/SAS for MATRICS-PASS_J; (Sumiyoshi et al., 2011a, 2011b) are currently under the standardization and validation.

International versions of these test batteries/scales are needed for the purpose of globalized multi-sites clinical trials for novel antipsychotic drugs. Also, advocacy of the MATRICS project seems to have promoted studies of functional outcome and its measurement world widely (Buchanan et al., 2005; Buchanan et al., 2011).

Despite an increasing interest in international versions of these neurocognitive and functional measures, the translations tend to be challenging both for developers and translators, as some tasks/items cannot be transferred straight forwardly, due to idiosyncrasies in languages, cultures, or socio-economical background (Velligan et al., 2012). Linguistic factors generally affect neuropsychological performance; a good example may be the Letter Number Sequence task in MCCB. The mental manipulation for sorting letters considerably differs between linearly sequenced alphabets and two-dimensionally arranged syllabic letters, such as Japanese $k a n a^{1}$. Apparently, the task demand is heavier in the latter, pointing to a need for some adjustments for the Japanese version.

Cultural factors, on the other hand, would affect the measurement of functional capacity or functional performance, as these measures are designed to be sensitive to social/daily living contexts. In UPSA-B, for instance, many countries do not have an equivalent schema for check payment which is included in the battery. Likewise, leisure activities (repertories, frequencies) in SFS and work status (i.e. paid workers, housewives/husbands) in Modified SFS/SAS for MATRIS-PASS would considerably vary across countries.

Those linguistic and cultural deficiencies could be controlled if the original developers or translators design equivalent counterparts and adjusting details (Sumiyoshi, 2011). In the Japanese version of UPSA-B (UPSA-B_J), for instance, the testing materials have been modified according to the Japanese socio-economical norms. Likewise, in the Japanese version of Modified SFS/SAS for MATRIS-PASS (Modified SFS/SAS for MATRIS-PASS_J), work section was adjusted to include house wife/husband category, so that the scale can cover a wider range of examinees.

\section{CONCLUSION}

The notion of functional outcome seems to have been well conceptualized (Green, 1996; Green et al., 2000) through the development of comprehensive neurocognitive batteries for patients with schizophrenia, especially MCCB. The spectrum of functional outcome, where neuropsychological performance is on the fundamental level while functional performance is on the high-end, may provide an incentive to include neurocognitive deficits into DSM diagnostic criteria for schizophrenia, which has emphasized functional status (Keefe et al., 2007).

For promoting the concept for functional outcome and advancing its measurement, several issues should be addressed. First, measures for functional performance have not been fully established yet. For example, currently, of the measures listed in Table 3, only two of them have been validated (Burns et al., 2007). Further effort should be directed concerning psychometric properties of functional performance scales. Second, although intervening variables may confound the ability of neuropsychological performance to predict social/community functioning, they may not be always regarded as 'nuisance' variables. Rather, some variables may help patients to achieve better social/community functioning if

\footnotetext{
${ }^{1}$ Fifty Japanese kanas are arranged two dimensionally, according to the vowels on the vertical axis and the consonants on the horizontal axis.
} 
they are positive ones, such as excellent system for social support in communities. The beneficial influence of intervening variables on functional outcome should be area for further investigation.

\section{ACKNOWLEDGEMENT}

This study was supported by Health and Labour Sciences Research Grants for Comprehensive Research on Disability, Health, and Welfare and grants-in-aid for Scientific Research from the Japan Society for the Promotion of Science. The authors would like to thank, Alan Milby, British Council Tokyo, for his helpful comments on the early version of the manuscript and proofreading the final draft.

\section{REFERENCES}

Birchwood, M., Smith, J., Cochrane, R., Wetton, S., \& Copestake, S. (1990). The Social Functioning Scale. The development and validation of a new scale of social adjustment for use in family intervention programmes with schizophrenic patients. British Journal of Psychiatry, 157, 853-859.

Buchanan, R. W., Davis, M., Goff, D., Green, M. F., Keefe, R. S., Leon, A. C., Nuechterlein, K. H., Laughren, T., Levin, R., Stover, E., Fenton, W., \& Marder, S. R. (2005). A summary of the FDA-NIMHMATRICS workshop on clinical trial design for neurocognitive drugs for schizophrenia. Schizophrenia Bulletin, 31, 5-19.

Buchanan, R. W., Keefe, R. S., Umbricht, D., Green, M. F., Laughren, T., \& Marder, S. R. (2011). The FDANIMH-MATRICS Guidelines for Clinical Trial Design of Cognitive-Enhancing Drugs: What Do We Know 5 Years Later? Schizophrenia Bulletin, 37, 1209-1217.

Burns, T., \& Patrick, D. (2007). Social functioning as an outcome measure in schizophrenia studies. Acta Psychiatry Scandinavia, 116, 403-418.

Couture, S. M., Penn, D. L., \& Roberts, D. L. (2006). The functional significance of social cognition in schizophrenia: a review. Schizophrenia Bulletin, 32 Suppl 1, S44-63.

Fanning, J. R., Bell, M. D., \& Fiszdon, J. M. (2012). Is it possible to have impaired neurocognition but good social cognition in schizophrenia? Schizophrenia Research, 135, 68-71.

Green, M. F. (1996). What are the functional consequences of neurocognitive deficits in schizophrenia? American Journal of Psychiatry, 153, 321-330.

Green, M. F. (2006). Cognitive impairment and functional outcome in schizophrenia and bipolar disorder. Journal of Clinical Psychiatry, 67 Suppl 9, 3-8; discussion 36-42.

Green, M. F., Bearden, C. E., Cannon, T. D., Fiske, A. P., Hellemann, G. S., Horan, W. P., Kee, K., Kern, R. S., Lee, J., Sergi, M. J., Subotnik, K. L., Sugar, C. A., Ventura, J., Yee, C. M., \& Nuechterlein, K. H. (2011). Social Cognition in Schizophrenia, Part 1: Performance Across Phase of Illness. Schizophrenia Bulletin.

Green, M. F., Kern, R. S., Braff, D. L., \& Mintz, J. (2000). Neurocognitive deficits and functional outcome in schizophrenia: are we measuring the "right stuff"? Schizophrenia Bulletin, 26, 119-136.

Green, M. F., Kern, R. S., \& Heaton, R. K. (2004). Longitudinal studies of cognition and functional outcome in schizophrenia: implications for MATRICS. Schizophrenia Research, 72, 41-51.

Green, M. F., Nuechterlein, K. H., Gold, J. M., Barch, D. M., Cohen, J., Essock, S., Fenton, W. S., Frese, F., Goldberg, T. E., Heaton, R. K., Keefe, R. S., Kern, R. S., Kraemer, H., Stover, E., Weinberger, D. R., Zalcman, S., \& Marder, S. R. (2004). Approaching a consensus cognitive battery for clinical trials in schizophrenia: the NIMH-MATRICS conference to select cognitive domains and test criteria. Biological Psychiatry, 56, 301-307.

Green, M. F., Nuechterlein, K. H., Kern, R. S., Baade, L. E., Fenton, W. S., Gold, J. M., Keefe, R. S., Mesholam-Gately, R., Seidman, L. J., Stover, E., \& Marder, S. R. (2008). Functional co-primary measures for clinical trials in schizophrenia: results from the MATRICS Psychometric and Standardization Study. American Journal of Psychiatry, 165, 221-228.

Green, M. F., Penn, D. L., Bentall, R., Carpenter, W. T., Gaebel, W., Gur, R. C., Kring, A. M., Park, S., Silverstein, S. M., \& Heinssen, R. (2008). Social cognition in schizophrenia: an NIMH workshop on definitions, assessment, and research opportunities. Schizophrenia Bulletin, 34, 1211-1220. 
Green, M. F., Schooler, N. R., Kern, R. S., Frese, F. J., Granberry, W., Harvey, P. D., Karson, C. N., Peters, N., Stewart, M., Seidman, L. J., Sonnenberg, J., Stone, W. S., Walling, D., Stover, E., \& Marder, S. R. (2011). Evaluation of Functionally Meaningful Measures for Clinical Trials of Cognition Enhancement in Schizophrenia. American Journal of Psychiatry.

Harvey, P. D., Green, M. F., \& Nuechterlein, K. H. (2010). Latest developments in the matrics process. Psychiatry (Edgmont), 7, 49-52.

Harvey, P. D., \& Velligan, D. I. (2011). International assessment of functional skills in people with schizophrenia.Innovations in Clinical Neuroscience 8, 15-18.

Horan, W. P., Green, M. F., Degroot, M., Fiske, A., Hellemann, G., Kee, K., Kern, R. S., Lee, J., Sergi, M. J., Subotnik, K. L., Sugar, C. A., Ventura, J., \& Nuechterlein, K. H. (2011). Social Cognition in Schizophrenia, Part 2: 12-Month Stability and Prediction of Functional Outcome in First-Episode Patients. Schizophrenia Bulletin.

Kaneda, Y., Sumiyoshi, T., Keefe, R., Ishimoto, Y., Numata, S., \& Ohmori, T. (2007). Brief assessment of cognition in schizophrenia: validation of the Japanese version. Psychiatry and Clinical Neurosciences, 61, 602-609.

Keefe, R. S., \& Fenton, W. S. (2007). How should DSM-V criteria for schizophrenia include cognitive impairment? Schizophrenia Bulletin, 33, 912-920.

Keefe, R. S., Goldberg, T. E., Harvey, P. D., Gold, J. M., Poe, M. P., \& Coughenour, L. (2004). The Brief Assessment of Cognition in Schizophrenia: reliability, sensitivity, and comparison with a standard neurocognitive battery. Schizophrenia Research, 68, 283-297.

Maruff, P., Thomas, E., Cysique, L., Brew, B., Collie, A., Snyder, P., \& Pietrzak, R. H. (2009). Validity of the CogState brief battery: relationship to standardized tests and sensitivity to cognitive impairment in mild traumatic brain injury, schizophrenia, and AIDS dementia complex. Archives of Clinical Neuropsychology, 24, 165-178.

McCrone, P., Thornicroft, G., Phelan, M., Holloway, F., Wykes, T., \& Johnson, S. (1998). Utilisation and costs of community mental health services. PRiSM Psychosis Study. 5. British Journal of Psychiatry, 173, 391-398.

Nemoto, T., Fujii, C., Miura, Y., Chino, B., Kobayashi, H., Yamazawa, R., Murakami, M., Kashima, H., \& Mizuno, M. (2008). Reliability and validity of the Social Functioning Scale Japanese version (SFS-J). Jpn Bull Soc Psychiat, 17, 188-196.

Nuechterlein, K. H., Green, M. F. (2006). MATRICS Consensus Cognitive Battery Manual: MATRICS Assessment Inc.

Pietrzak, R. H., Olver, J., Norman, T., Piskulic, D., Maruff, P., \& Snyder, P. J. (2009). A comparison of the CogState Schizophrenia Battery and the Measurement and Treatment Research to Improve Cognition in Schizophrenia (MATRICS) Battery in assessing cognitive impairment in chronic schizophrenia. Journal of clinical and experimental neuropsychology, 31, 848-859.

Subotnik, K. L., Nuechterlein, K. H., Kelly, K. A., Kupic, A. L., Brosemer, B., \& Turner, L. R. (2008). Modified Social Adjustment Scale-Work Outcome: Administration Manual: UCLA.

Sumiyoshi, C. (2011). Functional outcome in schizophrenia: Relation to the MATRICS consensus cognitive battery Jpn. J. Neuropsychopharmacol., 31, 251-257.

Sumiyoshi, C., \& Sumiyoshi, T. (2011a). Modified Social Adaptation Scale (Work Outcome)-Japanese version.

Sumiyoshi, C., \& Sumiyoshi, T. (2011b). Social Function Scale (MATRICS-PASS)-Japanese version.

Sumiyoshi, T., Sumiyoshi, C., \& Hemmi, C. (2011). UCSD Performance-Based Skills Assessment-Brief (UPSA-B): Japanese version; Administration and Scoring Manual

Velligan, D. I., Rubin, M., Fredrick, M. M., Mintz, J., Nuechterlein, K. H., Schooler, N. R., Jaeger, J., Peters, N. M., Buller, R., Marder, S. R., \& Dube, S. (2012). The cultural adaptability of intermediate measures of functional outcome in schizophrenia. Schizophrenia Bulletin, 38, 630-641.

Yoshida, T., Suga, M., Arima, K., Muranaka, Y., Tanaka, T., Eguchi, S., Lin, C., Yoshida, S., Ishikawa, M., Higuchi, Y., Seo, T., Ueoka, Y., Tomotake, M., Kaneda, Y., Darby, D., Maruff, P., Iyo, M., Kasai, K., Higuchi, T., Sumiyoshi, T., Ohmori, T., Takahashi, K., \& Hashimoto, K. (2011). Criterion and construct validity of the CogState Schizophrenia Battery in Japanese patients with schizophrenia. PLoS One, 6, e20469. 